\title{
A Simple and Effective Microwave Invisibility Cloak Based on Frequency Selective Surfaces
}

Pedro Yuste ${ }^{1}$, Juan M. Rius ${ }^{1}$, Jordi Romeu ${ }^{1}$, Sebastián Blanch Boris ${ }^{1}$, Alexander Heldring ${ }^{1}$, Eduard Ubeda ${ }^{1}$

${ }^{1}$ AntennaLAB, Department of Signal Theory and Communications, Universitat Politcnica de Catalunya (BarcelonaTECH),

Barcelona, Spain, rius@tsc.upc.edu

\begin{abstract}
This paper presents the design, simulation, manufacturing and testing of a simple invisibility cloak based on a frequency selective surface (FSS). The work is focused on cloaking an electrically thin dielectric cylinder with an easy to manufacture FSS made of copper strips glued to the cylinder surface. In contrast to many papers in the literature, the full procedure from formulation to measurement results is presented here. An original approach to obtain the effective surface impedance of the cylindrical FSS from either simulated or measured far fields is introduced. The measurement results show excellent and relatively wide band performance of the cloak prototype.
\end{abstract}

Index Terms-metamaterials, cloaking, mantle cloaks, scattering cancellation, frequency selective surfaces.

\section{INTRODUCTION}

In recent years several invisibility cloaking techniques have been developed. Some of them, like transformation-based, carpet or plasmonic cloaking techniques have important handicaps, such as large losses, difficult implementation or very narrow bandwidth. In order to easily build a cloak using cheap equipment, the scattering cancellation technique with a Frequency Selective Surface (FSS) as a mantle cloak is very attractive [2].

An FSS is probably the cheapest and easiest to implement choice to put scattering cancellation into practice. The electromagnetic behavior of FSS can be described by means of a surface impedance, henceforth $Z_{s}$, which relates the averaged tangential electric field on the surface to the averaged induced electric current density in this way [3]:

$$
\begin{gathered}
\vec{E}^{\tan }=Z_{s} \cdot \vec{J}_{s} \\
\hat{n} \times\left(\vec{H}_{t a n}^{+}-\vec{H}_{\text {tan }}^{-}\right)=\vec{J}_{s}
\end{gathered}
$$

The geometry of an FSS consisting of a very thin metallic layer can be designed to satisfy the average surface impedance $Z_{s}=R_{s}-j X_{s}$ that minimizes scattering. Moreover, at radio frequencies the FSS can be assumed lossless and therefore its average surface impedance is purely reactive, $Z_{s}=-j X_{s}$.

\section{Mantle Cloak Scattering Cancellation}

An incoming plane wave propagating along the $\mathrm{x}$-axis and impinging upon a circular cylinder $\vec{E}^{i n c}=\hat{z} E_{0} e^{-j k_{0} x}$ can be

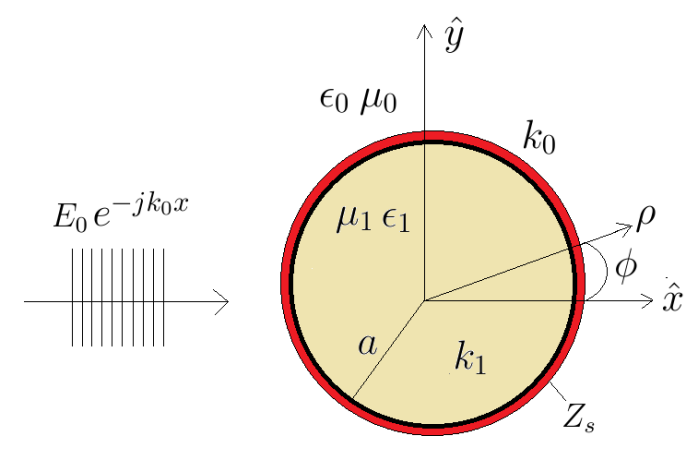

Fig. 1. Geometrical layout of the cylinder and the cloak (marked in red).

expressed in cylindrical coordinates as [4]:

$$
\vec{E}^{i n c}=\hat{z} E_{0} e^{-j k_{0} \rho \cos \phi}=\hat{z} E_{0} \sum_{n=-\infty}^{\infty} j^{-n} J_{n}\left(k_{0} \rho\right) e^{j n \phi}
$$

The cylindrical wave expressions of the scattered field outside the dielectric cylinder and the total field inside are respectively:

$$
\begin{aligned}
\vec{E}^{s} & =\hat{z} E_{0} \sum_{n=-\infty}^{\infty} a_{n} H_{n}^{(2)}\left(k_{0} \rho\right) e^{j n \phi} \\
\vec{E}^{i} & =\hat{z} E_{0} \sum_{n=-\infty}^{\infty} b_{n} J_{n}\left(k_{1} \rho\right) e^{j n \phi}
\end{aligned}
$$

In the scenario represented in Figure $1, \mathrm{Zs}$ is the surface impedance of the cloak, and therefore the boundary condition at $\rho=a$ becomes:

$$
\hat{n} \times\left.\left(H_{\phi}^{\text {out }}-H_{\phi}^{\text {in }}\right) \hat{\phi}\right|_{\rho=a}=\left.J_{z} \hat{z}\right|_{\rho=a}=\left.\frac{E_{z}}{Z_{s}}\right|_{\rho=a}
$$

where both magnetic fields can be obtained by applying the Maxwell-Faraday Equation.

Therefore, since $Z_{s}\left(H_{\phi}^{\text {out }}-H_{\phi}^{\text {in }}\right)=E_{z}$, the following system of equations, combining (4), (5) and (6), arises:

$$
\left(\begin{array}{cc}
H_{n}^{(2)}\left(k_{0} a\right) & -J_{n}\left(k_{1} a\right) \\
\frac{1}{j \eta_{0}} Z_{s} H_{n}^{(2)^{\prime}}\left(k_{0} a\right) & -\frac{1}{j \eta_{1}} Z_{s} J_{n}^{\prime}(k 1 a)-J_{n}\left(k_{1} a\right)
\end{array}\right)\left(\begin{array}{l}
a_{n} \\
b_{n}
\end{array}\right)=
$$



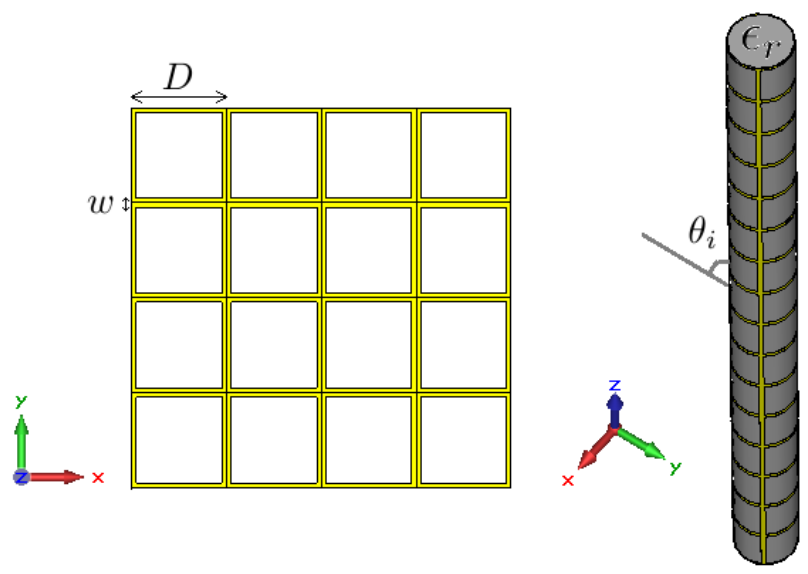

Fig. 2. Mesh-grid geometry.

$$
\begin{gathered}
=\left(\begin{array}{c}
-j^{-n} J_{n}\left(k_{0} a\right) \\
-j^{-n} \frac{1}{j \eta_{0}} Z_{s} J_{n}^{\prime}\left(k_{0} a\right)
\end{array}\right) \\
a_{n}=\frac{j^{-n}\left|\begin{array}{cc}
-J_{n}\left(k_{0} a\right) & -J_{n}\left(k_{1} a\right) \\
-Z_{s} J_{n}^{\prime}\left(k_{0} a\right) & -n_{1} Z_{s} J_{n}^{\prime}\left(k_{1} a\right)-j \eta_{0} J_{n}\left(k_{1} a\right)
\end{array}\right|}{\left|\begin{array}{cc}
H_{n}^{(2)}\left(k_{0} a\right) & -J_{n}\left(k_{1} a\right) \\
Z_{s} H_{n}^{(2)^{\prime}}\left(k_{0} a\right) & -n_{1} Z_{s} J_{n}^{\prime}\left(k_{1} a\right)-j \eta_{0} J_{n}\left(k_{1} a\right)
\end{array}\right|},
\end{gathered}
$$

where $n_{1}=\frac{\eta_{0}}{\eta_{1}}=\sqrt{\frac{\epsilon_{1}}{\epsilon_{0}}}$.

The $a_{n}$ coefficients must be minimized in order to reduce scattering in equation (4). In case of electrically thin cylinders ( $a<<\lambda$ and dominant term $n=0$ ) the condition $a_{0}=0$ is imposed to find out the surface impedance

$$
Z_{s}=j X_{s}=\frac{j \eta_{0} J_{0}\left(k_{0} a\right) J_{0}\left(k_{1} a\right)}{n_{1} J_{0}\left(k_{0} a\right) J_{1}\left(k_{1} a\right)-J_{1}\left(k_{0} a\right) J_{0}\left(k_{1} a\right)}
$$

which is the electrical reactance that the surface impedance of an FSS attached to a thin cylinder must have in order to achieve the best possible cancellation.

By using a mesh-grid design, the one that has been chosen in this work, the cell surface impedance $Z_{s}$ can be modeled as [5]:

$$
Z_{s}^{T M}=\frac{j \omega \eta_{0} D}{2 c \pi} \ln \left(\csc \left(\frac{\pi w}{2 D}\right)\right)\left(1-\frac{\sin ^{2} \theta_{s}}{\epsilon_{r}+1}\right)
$$

in the TM-polarized case.

\section{SimUlATION}

The cylinder to be cloaked is made of PVC, whose relative permittivity is $\epsilon_{r}=2.723$, as measured experimentally. It is $33.6 \mathrm{~cm}$ long with a radius $a=1.34 \mathrm{~cm}$. The incident field is a plane wave that impinges perpendicularly to the cylinder axis $\left(\theta_{i}=\frac{\pi}{2}\right)$.

The required cloak impedance to cancel $a_{0}$ at $f_{0}=$ $3.75 \mathrm{GHz}$ is $Z_{s}=j 195.96 \Omega$. The mesh grid cell dimensions

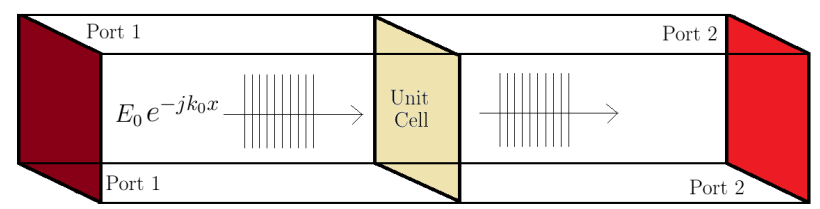

$\vec{I} \rightarrow$

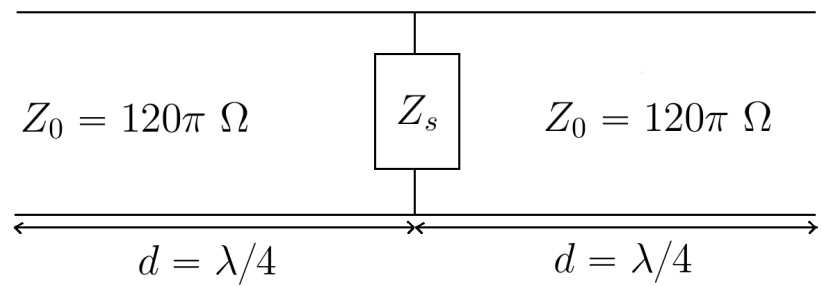

Fig. 3. Unit cell system considered as a transmission line with a parallel impedance Zs.

that lead to this FSS impedance are: period $D=0.021 \mathrm{~mm}$ and strip width $w=0.0019 \mathrm{~mm}$.

\section{A. Infinite Planar FSS Impedance}

Assuming an infinite planar periodic FSS, by means of Floquet Mode Theory one can deduce the behavior of the full (infinite) structure from the simulation of a single cell composing it. Computer Simulation Technology software (CST) [6] has been used with two Floquet ports at both sides of an infinite mesh-grid structure. The FSS impedance $Z_{s}$ is computed from the S-Parameters using a transmission line model as depicted in figure 3,

$$
Z_{s}=-Z_{0}\left(\frac{1+S_{11 \text { cell }}}{2 S_{11 \text { cell }}}\right)
$$

where $S_{11 \text { cell }}=S_{11 \text { port }} \cdot e^{\frac{j 2 \pi d}{\lambda}}, S_{11 \text { port }}$ is the reflection coefficient computed by CST at the Floquet port and $d$ is the length of the transmission line from the Floquet port to the plane of the FSS. Figure 4 shows the simulated FSS surface impedance for the proposed mesh-grid cell.

As expected, not only the real part is zero at all frequencies $\left(Z_{s}\right.$ is purely reactive in the lossless case) but also the surface impedance which is obtained around the design frequency $\left(Z_{s}=184.4 \Omega\right)$ is very close to the desired one $\left(Z_{s}=195.96 \Omega\right)$.

\section{B. Cylindrical FSS impedance}

The entire cylindrical cloak structure has been meshed and simulated in FIESTA-3D (Fast Integral Equation Solver for scaTterers and Antennas in 3D) [7], a Method of Momentsbased software developed at the AntennaLAB of the Universitat Politècnica de Catalunya.

One can take advantage of the fact that the cylindrical expansion coefficient $a_{0}$ and the averaged scattered far field $E^{s}(\phi)$ at constant $\rho$ are closely related to each other to find out the corresponding surface impedance: 


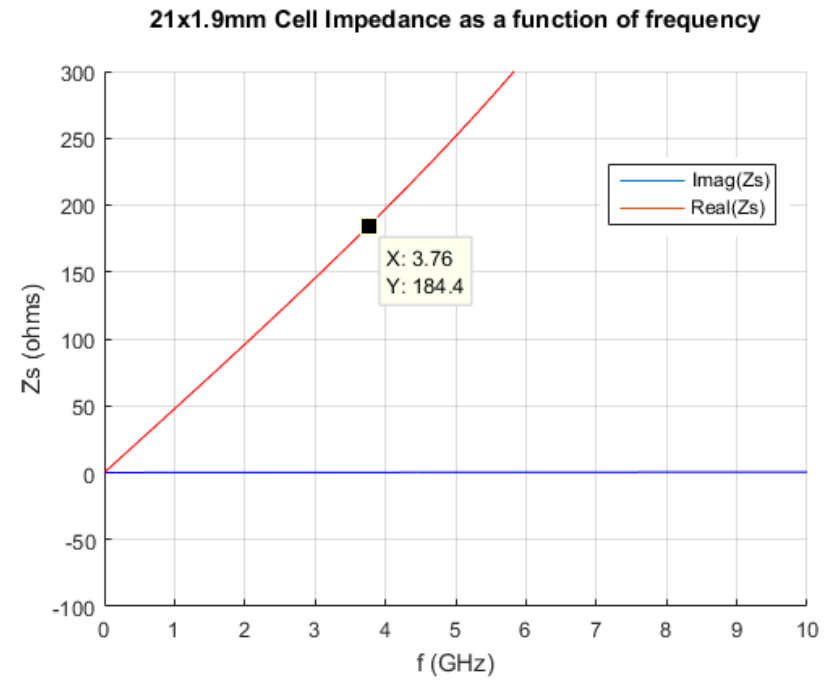

Fig. 4. Planar infinite FSS cell impedance as a function of frequency for mesh-grid cells.

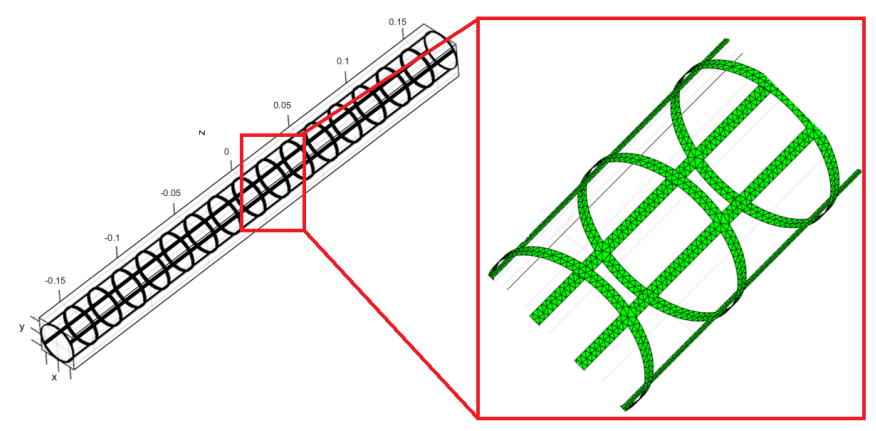

Fig. 5. Mesh-grid cloaking structure and zoomed mesh view.

$$
a_{0} H_{0}^{(2)}\left(k_{0} \rho\right)=\frac{1}{2 \pi} \int_{0}^{2 \pi} E_{z}^{2 D T M}(\phi) d \phi
$$

where $E_{z}^{2 D T M}$ is the scattered field of the infinite cylinder 2D problem with TM polarization.

Using the large argument approximation of the Hankel function

$$
H_{n}^{(2}\left(k_{o} \rho\right) \approx \sqrt{\frac{2}{\pi k_{0} \rho}} e^{-j\left[k_{0} \rho-n \frac{\pi}{2}-\frac{\pi}{4}\right]}
$$

and the approximate far field relation for the $2 \mathrm{D}$ and $3 \mathrm{D}$ problems

$$
E_{z}^{2 D T M} \approx \frac{\sqrt{\lambda \rho}}{L} e^{-j \pi / 4} E_{z}^{3 D}
$$

where $E_{z}^{3 D}=E^{s}$ is the far field scattered by a cylinder of length $L$ and perpendicularly incident plane wave, we find that the cylindrical expansion coefficient $a_{0}$ is related to the average of the 3D scattered far field $E^{s}(\phi)$ at a constant $\rho$ as:

$$
a_{0}=\frac{j}{2 L} \int_{0}^{2 \pi} E^{s}(\phi) d \phi
$$

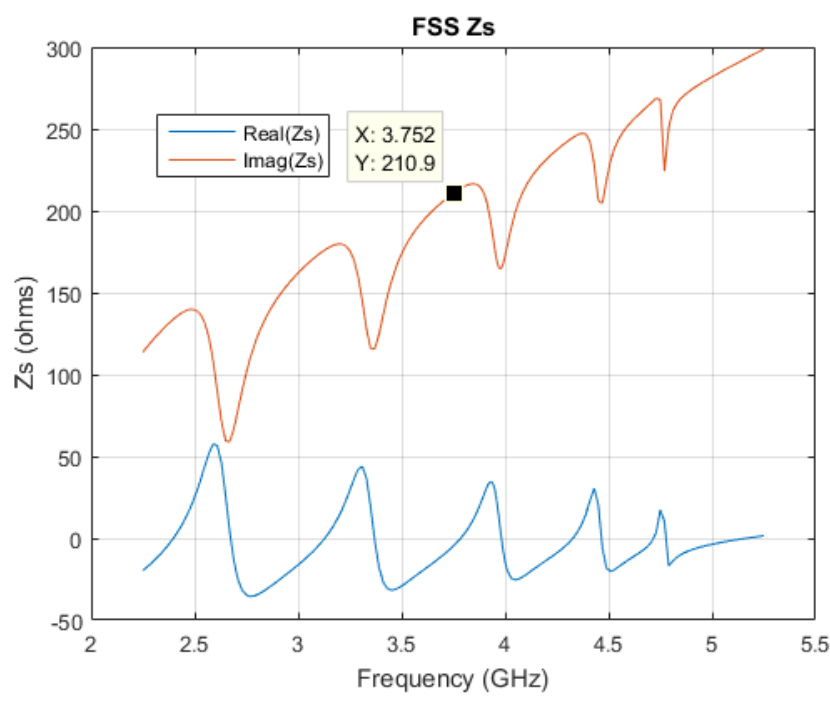

Fig. 6. Simulated $Z_{s}$ of the cloak (no cylinder inside) as a function of frequency.

Now, isolating $Z_{s}$ in (8) we can find the effective surface impedance of the cylindrical FSS:

$$
Z_{s}=\frac{j \eta_{0}\left(a_{0} H_{00} J_{01}+J_{00} J_{01}\right)}{a_{0} n_{1} H_{00} J_{11}-a_{0} H_{10} J_{01}+n_{1} J_{00} J_{11}-J_{01} J_{10}}
$$

where:

- $J_{00}=J_{0}\left(k_{0} a\right), J_{01}=J_{0}\left(k_{1} a\right), J_{10}=J_{1}\left(k_{0} a\right)$ and $J_{11}=J_{1}\left(k_{1} a\right)$

- $H_{00}=H_{0}^{(2)}\left(k_{0} a\right)$ and $H_{10}=H_{1}^{(2)}\left(k_{0} a\right)$

Figure 6 shows the evolution of the surface impedance as a function of frequency. Comparing the results in Figure 4 and 6 , we can assess the difference between the $Z_{s}$ of the ideal infinite planar FSS and the cylindrical one.

At the design frequency $(3.75 \mathrm{GHz})$ the surface impedance takes a value of $Z_{s}=j 210.7 \Omega$, whereas the desired impedance $Z_{s}=j 194.9 \Omega$ occurs at $3.61 \mathrm{GHz}$. It is also important to note that this surface impedance clearly behaves as expected since the real part converges at $\operatorname{Real}\left(Z_{s}\right)=0 \Omega$ (beyond $5 \mathrm{GHz}$ ) and the imaginary part follows the same asymptotic trend as in Figure 4.

\section{Full-wave Simulation of Mantle Cloak}

Two different simulations, one over the bare cylinder and another over the cloaked one (cylinder+cloak) have been computed with FIESTA-3D software in order to assess cloaking performance. Figure 7 shows the far fields integrated for $\phi$ angles around the cylinder and 8 shows the cloak attenuation. The red line corresponds to the FIESTA-3D simulation results, while the blue one is the theoretical result from $2 \mathrm{D}$ cylindrical expansion coefficients (4), (8) using $Z_{s}=j 195 \Omega$ to set $a_{0}=0$ at the frequency of design $(3.75 \mathrm{GHz})$.

According to the simulation results (red line), almost a $30 \mathrm{~dB}$ reduction is obtained, even though theoretically it should be $-68 d B$ (in blue). This is an excellent result with a 


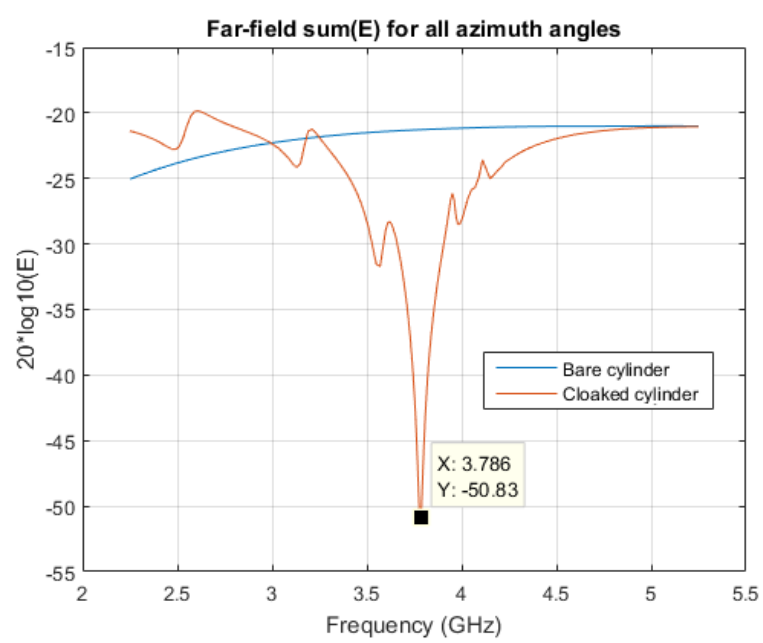

Fig. 7. Integration of $E^{s}(\phi)$ for all $\phi$ angles as a function of frequency in case of bare and cloaked cylinders.

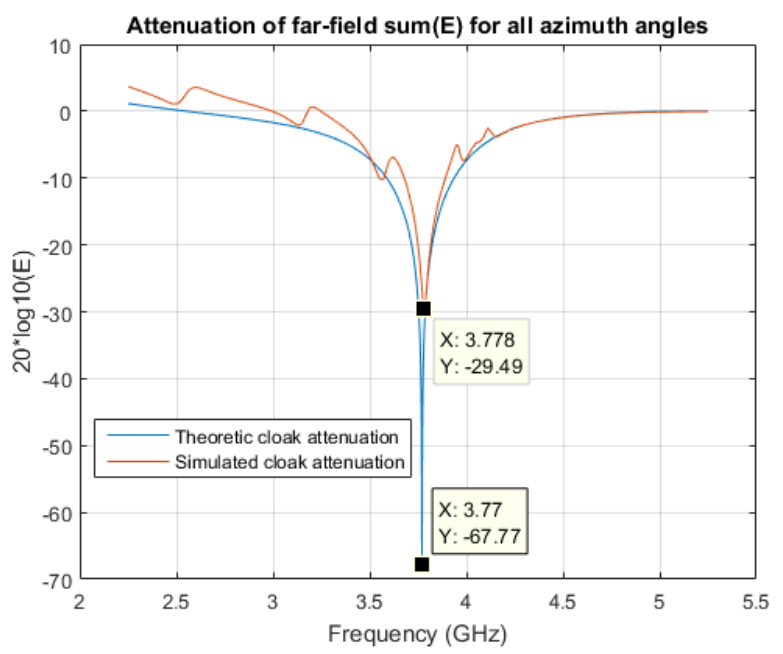

Fig. 8. Cloaking attenuation: simulated and theoretical.

view to future practical implementations, albeit the peak is not centered exactly where desired $(3.75 \mathrm{GHz})$ but at $3.786 \mathrm{GHz}$.

The cloaking effect can be also observed analyzing the radiation pattern at two contrasting frequencies, which is depicted in Figure 9.

\section{Manufacturing AND Experimental Testing}

The cloak was firstly conceived as a $32 \times 4$ cell copper sheet, like the one simulated in either CST or FIESTA-3D. However, due to the extremely thinness of the copper sheet metal $(70 \mu \mathrm{m})$ some of the usual manufacturing methods were discarded, such as milling machines or laser cutters. Finally, chemical etching by using a photoplotter was revealed as a feasible way to obtain a suitable prototype. The plastic film and the copper cloak after the chemical process are shown in Fig. 10. As a consequence of some technical limitations of the equipment, manufacturing the whole $32 \times 4$ cell cloak was

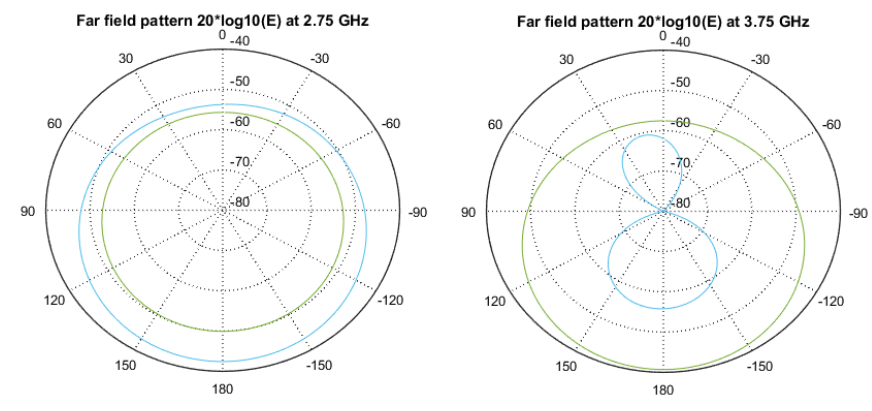

Fig. 9. E-field radiation patterns for the non-cloaked (in green) and the cloaked case (in blue) at two frequencies: one away from design $(2.75 \mathrm{GHz}$, on the left) and another one at the design frequency (3.75 $\mathrm{GHz}$, on the right).
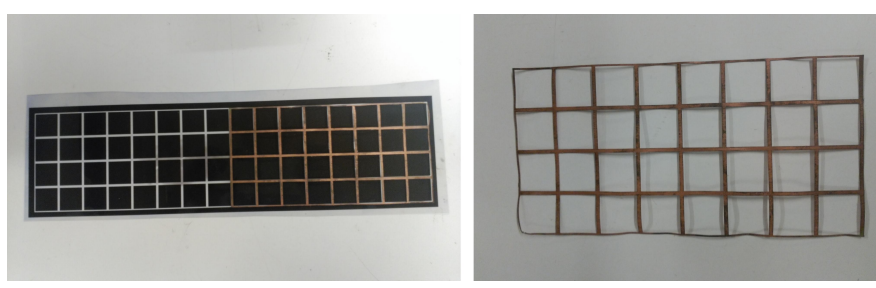

Fig. 10. Black-and-white plastic film mask, basically a negative image, to print the design on the copper sheet metal (left) and final resulting section of cloak (right). Notice that it perfectly fits in the plastic film, what confirms the good accuracy of the procedure.

impossible and four different sections had to be printed for a further assembly. Finally, the FSS structure was glued to the curved surface of the dielectric cylinder.

\section{A. Experimental Setup and Scattering Results}

The bistatic measurement setup is shown in Fig. 11. In order to integrate the scattered fields for $\phi$ angles, the receiver antenna has to be progressively moved to different positions around the cylinder. Due to space limitations in the anechoic chamber when setting the equipment, the radiation pattern has been assumed symmetric and only five angles covering half a circumference $\left(0^{\circ}, 45^{\circ}, 90^{\circ}, 135^{\circ}\right.$ and $\left.180^{\circ}\right)$ are added up.

Figure 12 shows the summation of all measurements at different angular positions. The cloaking performance of the prototype is excellent, but the peak is located at $3.39 \mathrm{GHz}$ instead of the design frequency $(3.75 \mathrm{GHz})$. The the most likely cause for this shift lies in the lack of accuracy during the manufacturing process, since extraordinary precision is required to make a prototype perfectly fitting the theoretical model. Nevertheless, even though the peak has been shifted, these results still agree well with theory and simulation. The bandwidth of the order of $100 \mathrm{MHz}$ is remarkable.

\section{CONClusion}

The design of a simple and effective mantle cloak based on a frequency selective surface has been presented. Simulation and experimental prototype measurements show excellent attenuation and relatively wide band results. An original approach to obtain the effective surface impedance of the cylindrical 

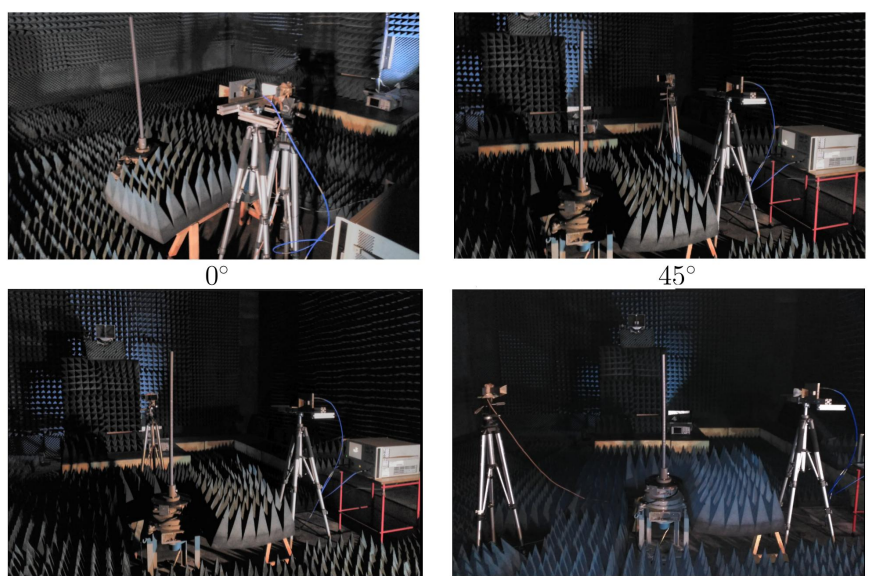

90

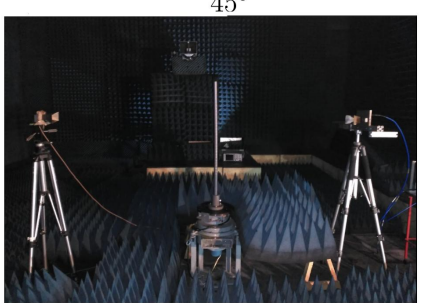

$180^{\circ}$

Fig. 11. Measurement set-up at four different angles.

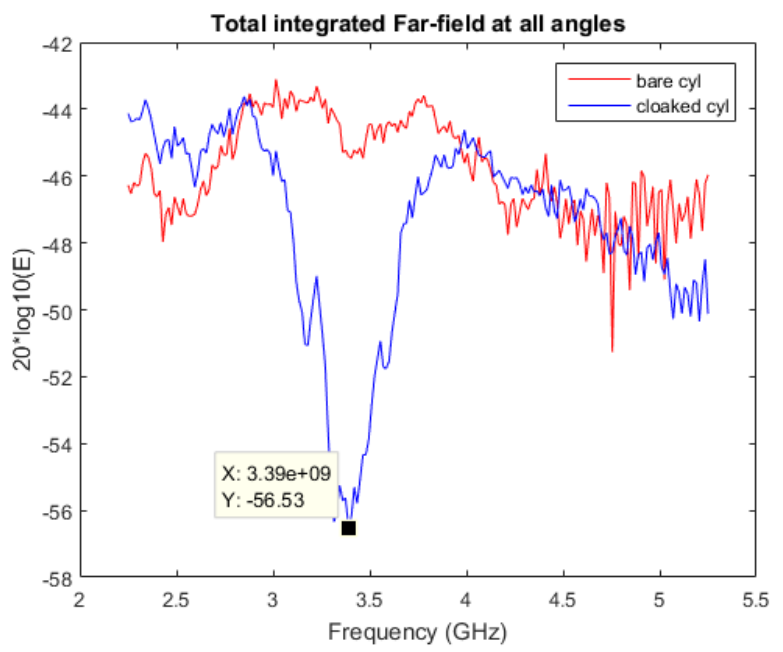

Fig. 12. Total E-Field calculated by adding all the angular measurements.

FSS from either simulated or measured far fields has been introduced.

\section{ACKNOWLEDGMENT}

This work has been supported by the Spanish "Comisión Interministerial de Ciencia y Tecnología" (CICYT) under projects TEC2013-47360-C3-1-P and TEC2016-78028-C3-1P.

\section{REFERENCES}

[1] Yuste Gallego, Pedro; Rius Casals, Juan Manuel; Romeu Robert, Jordi, Microwave Invisibility Cloaks based on Frequency Selective Surfaces, Telecomunication Engineering Master Thesis at Universitat Politcnica de Catalunya, Barcelona, September 2016.

[2] Monti, Alessio; Soric, Jason; Al, Andrea; Toscano, Alessandro; Bilotti, Filiberto, Anisotropic Mantle Cloaks for TM and TE Scattering Reduction, IEEE Transactions on Antennas and Propagation, Vol.63, No.4, pp. 1775, April 2015.

[3] Chen, Pai-Yen; Soric, Jason; Al, Andrea, Invisibility and Cloaking Based on Scattering Cancellation, Advanced Materials, Volume 24, Issue 44 pp. 281-304, November 20, 2012.
[4] Balanis, Constantine A., Advanced Engineering Electromagnetics, Appendix $I V$, p. 603, Arizona State University, published by John Wiley and Sons, 1989.

[5] R. Padooru, Yashwanth; B. Yakolev, Alexander; Chen, Pai-Yen; Al, Andrea, Analytical modeling of conformal mantle cloaks for cylindrical object using sub-wavelength printed and slotted arrays, Journal of Applied Physics, Vol. 112, 034907 (2012).

[6] Computer Simulation Technology, http://www.cst.com

[7] J.M. Rius, J. Parrn, A. Heldring, J.M. Tamayo and E. Ubeda, Fast Iterative Solution of Integral Equations with Method of Moments and Matrix Decomposition Algorithm Singular Value Decomposition, IEEE Trans. on Antennas and Propagation, special issue on Large and Multiscale Computational Electromagnetics, Vol. 56, No. 8, pp. 2314-2324, August 2008. 